\title{
Preparation and Characterization of Activated Alumina
}

\author{
A R. Rabia ${ }^{1 * *}, A . H$. Ibrahim $^{1}$, and N.N. Zulkepli ${ }^{2}$ \\ ${ }^{1}$ School of Environmental Engineering, Universiti Malaysia Perlis, Kompleks Pusat Pengajian Jejawi \\ 3, 02600 Arau, Perlis, Malaysia \\ ${ }^{2}$ Faculty of Engineering Technology, Kampus Uniciti Alam Sg. Chuchuh, 02100 Padang Besar, \\ Universiti Malaysia Perlis (UniMAP)
}

\begin{abstract}
Activated alumina is a high surface area and highly porous form of aluminum oxide that can be employed for contaminant species adsorb from ether gases or liquids without changing its form. The research in getting this material has generated huge interested. Thus, this paper presented preparation of activated alumina from chemical process. Pure aluminum (99.9\% pure) reacted at room temperature with an aqueous $\mathrm{NaOH}$ in a reactor to produce a solution of sodium aluminate $\left(\mathrm{NaAlO}_{2}\right)$. This solution was passed through filter paper and the clear filtrate was neutralized with $\mathrm{H}_{2} \mathrm{SO}_{4}$, to $\mathrm{pH} 6,7$ or 8 , resulting in the precipitation of a white gel, $\mathrm{Al}(\mathrm{OH}) 3 \cdot \mathrm{XH} 2 \mathrm{O}$. The washed gel for sulfate ions were dried at $80{ }^{\circ} \mathrm{C}$ for $6 \mathrm{~h}$, a 60 mesh sieve was to separate and sort them into different sizes. The samples were then calcined (burn) for $3 \mathrm{~h}$ in a muffle furnace, in air, at a heating rate of $2{ }^{\circ} \mathrm{C} \mathrm{min}^{-1}$. The prepared activated alumina was further characterized for better understanding of its physical properties in order to predict its chemical mechanism.
\end{abstract}

\section{Introduction}

The water treatment technology via aluminum has gained a great importance in recent time competing with conventional technologies in various industrial fields in particular for chemical and petrochemical industries that material have shown capability to treat water long time in history [1]. Currently, Aluminum play a special role in the field of energy and electric power as one of the elemental parts of conducting material due to availability and cheapness that material has gain a wider application, it has be use through the with help addition component such membrane, use in separation process technology which appeared [2-5]. Since the beginning of 1960 's, aluminum was used as coagulant in local water purification. At the beginning of 1970's, the first industrial installation of membrane using aluminum was initiated and using this platform, the water purification related activities rapidly grown and developed large-scale commercial [6]. With this initiative, it began to insert significant improvements in membrane selectivity / permeability and process design $[3]$.

\footnotetext{
*Corresponding author: elmahdy1999@yahoo.com
} 
The main goal of improving the membranes permeability is to achieve constant quality, high performance membrane and systems for desired applications with enhanced troubleshooting capabilities [7]. Moreover, based on this membrane development, the removal of contaminate from in water is taking great interest in the last decades from the environmental and energy perspective, respectively [8]. From the environmental view point, heavy metals considered one of the major element which causes a pollution in the water and the environment as well as being a main reason of the health issue because its increased concentrations of the this element due to the wide use of the industrial process as a main source of heavy metal [9]. While from the health perspective, metal heavy is one of the most pollutants in the feeding of natural environment and therefore must be removed because it subsequently lead to reduction of the bring health problem [10].

The alumina based technology in addition to what has been mentioned, its can equally play a major role in making water purification processes economically feasible. Today, treatment with aluminum are a promising, effective and reliable solution when compared to the traditional process in terms of effective cost, preserving environmental and capability to remove contaminants such as carbon dioxide and hydrogen sulfide across wide range of operating conditions [11]. The process of forming good treatment depends mainly on the selection of appropriate materials meaning making aluminum active and is one of the key factors to produce a good treatment and the success of the performance process. In general, material prepared which consists of polymer, solvent and non-solvent using are the major techniques to produce asymmetric membrane structural [12].

For the membrane fabrication technique, the mechanism to invert phase is introduced by called phase inversion and subsequent immersion precipitate process is a proposed and well-known approach for making polymeric based membranes device [13]. However, this is not well promising like activated alumina. By the activated aluminum with the immersion of a designed substrate in pre-coagulation bath and solvent in the solution is transferred with a non-solvent in the precipitating media and phase thus, phase inversion occurs [14]. Good number of research communities has tried to attempt to give the explanation on the membrane-forming and development mechanism during and after the phase inversion process [14-18].

For instance, in membrane fabrication, addition of non-solvent additive into activated alumina formulation might be useful due to its influence to physical properties of membrane such as pore formation, pore distribution by suppression of macro voids or micro voids evolution [19-23].

\section{Materials and Methods}

The samples of aluminum powder was obtained in Meridian World Sdn. Bhd, which is one of the environmental service providing company at Sungai Petani, Kedah Darul Aman, Malaysia. Pure aluminum (99.9\% pure), reacted, at room temperature, with a aqueous $\mathrm{NaOH}$ in a reactor to produce a solution of sodium aluminate $\left(\mathrm{NaAlO}_{2}\right)$. This solution was passed through filter paper and the clear filtrate was neutralized with $\mathrm{H}_{2} \mathrm{SO}_{4}$, to $\mathrm{pH} 6,7$ or 8 , resulting in the precipitation of a white gel, $\mathrm{Al}(\mathrm{OH})_{3} \cdot \mathrm{XH}_{2} \mathrm{O}$. The gel was washed until no more sulfate ions were detected in the washings. Finally, they were dried at $80{ }^{\circ} \mathrm{C}$ for 6 $\mathrm{h}$, then ground and passed through a 60 mesh sieve. The samples were then calcined (burn) for $3 \mathrm{~h}$ in a muffle furnace, in air, at a heating rate of $2{ }^{\circ} \mathrm{C} \mathrm{min}^{-1}$. Finally activated alumina was obtained. 


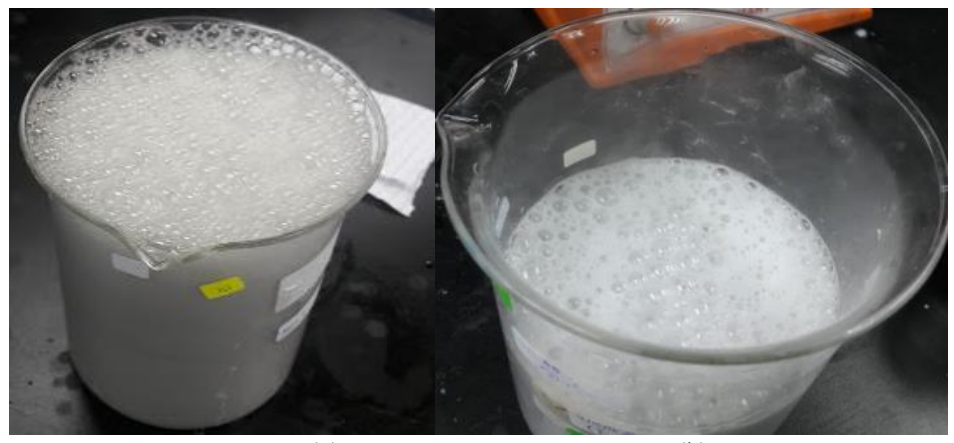

(a)

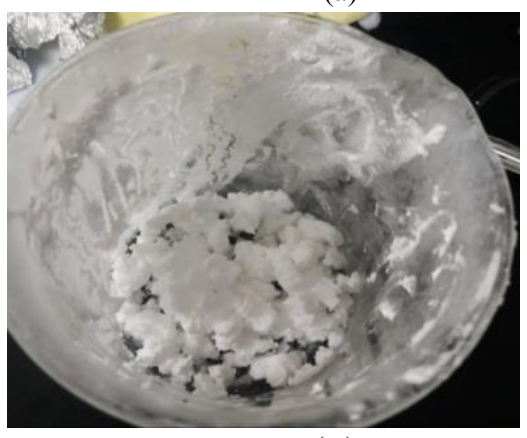

(c) (b)

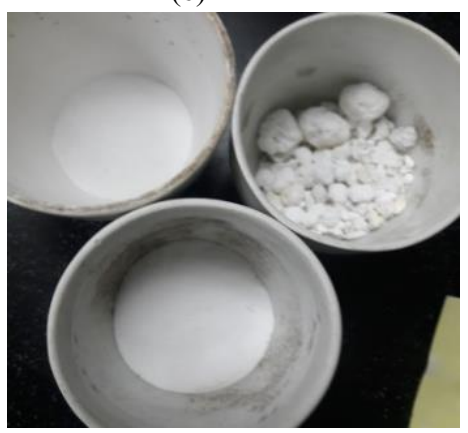

(d)

Fig.1. Show the prepared alumina (a) Bubbles (b) Bubbles settled (c) Slake alumina (d) final process for the alumina preparation.

The prepared alumina via the pure aluminum (99.9\% pure) reaction was observed during proves Fig. 1 shown the bubbles raising during the reaction, from the Fig. $1 \mathrm{~b}$, the bubbles settle down at room temperature, with a aqueous $\mathrm{NaOH}$ in a reactor to produce a solution of sodium aluminate $\left(\mathrm{NaAlO}_{2}\right)$. This solution was passed through filter paper and the clear filtrate was neutralized with $\mathrm{H}_{2} \mathrm{SO}_{4}$, to $\mathrm{pH} 6,7$ or 8, resulting in the precipitation of a white gel, $\mathrm{Al}(\mathrm{OH})_{3} \cdot \mathrm{XH}_{2} \mathrm{O}$. The gel was washed until no more sulfate ions were detected in the washings, which were observed in Fig. 3 and 4. Finally, they were dried at $80{ }^{\circ} \mathrm{C}$ for $6 \mathrm{~h}$, then ground and passed through a 60 mesh sieve. The samples were then steadily calcined (burn) to remove other unwanted for $3 \mathrm{~h}$ in a muffle furnace, in air, at a heating rate of $2{ }^{\circ} \mathrm{C} \mathrm{min}^{-1}$ as shown in Fig. 1c and $1 \mathrm{~d}$.

\section{Results and discussions}

The Fig. 2 shown the spectrum of alumina, however, EDX spectrum shows that the existence of oxygen (oxide) and chloride. This is happening due to the diffusion between the oxide layers, which is located underneath the alumina. When the diffusion takes place, the oxygen may exist. An investigated was equally conducted on the present of prepared alumina structures based on EDX. Fig. 2 shows the of highest peak of the alumina structures. The alumina exhibits a homogeneous size over the whole substrate, uniform distribution of the might be expected on structures since show only 1 peak as can be observed. 


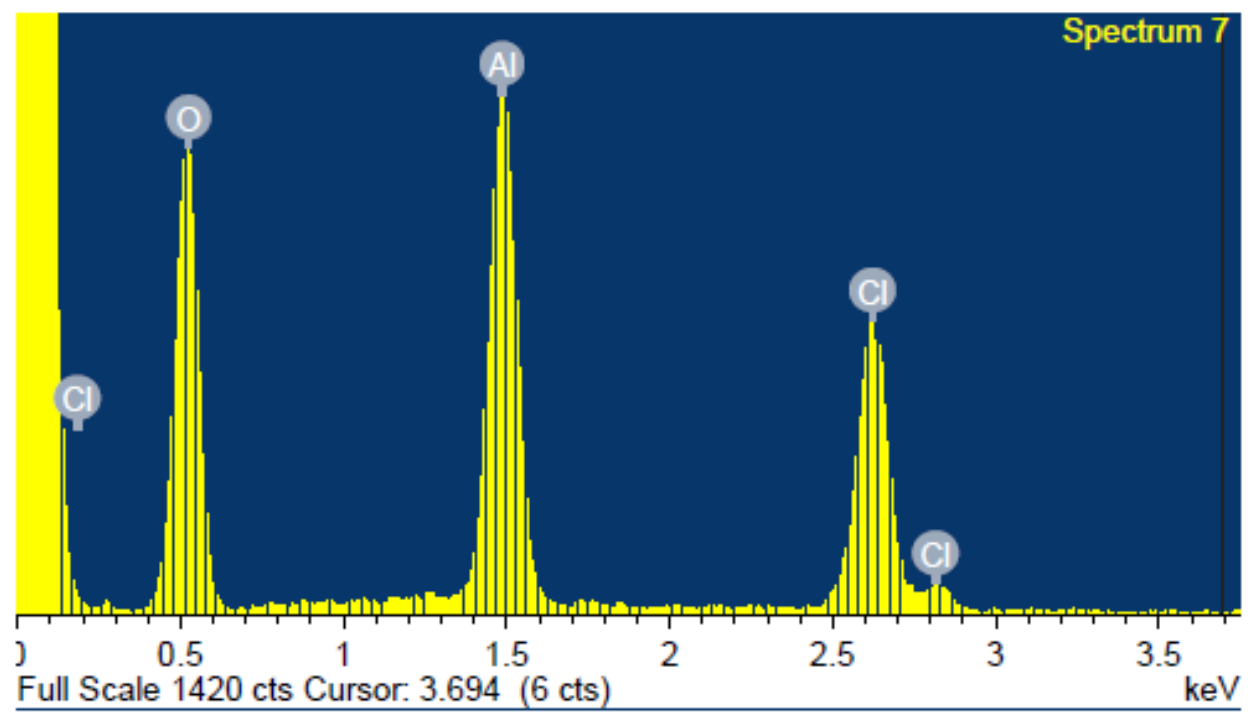

Fig. 2. Cross section of alumina highlighted with X35000.

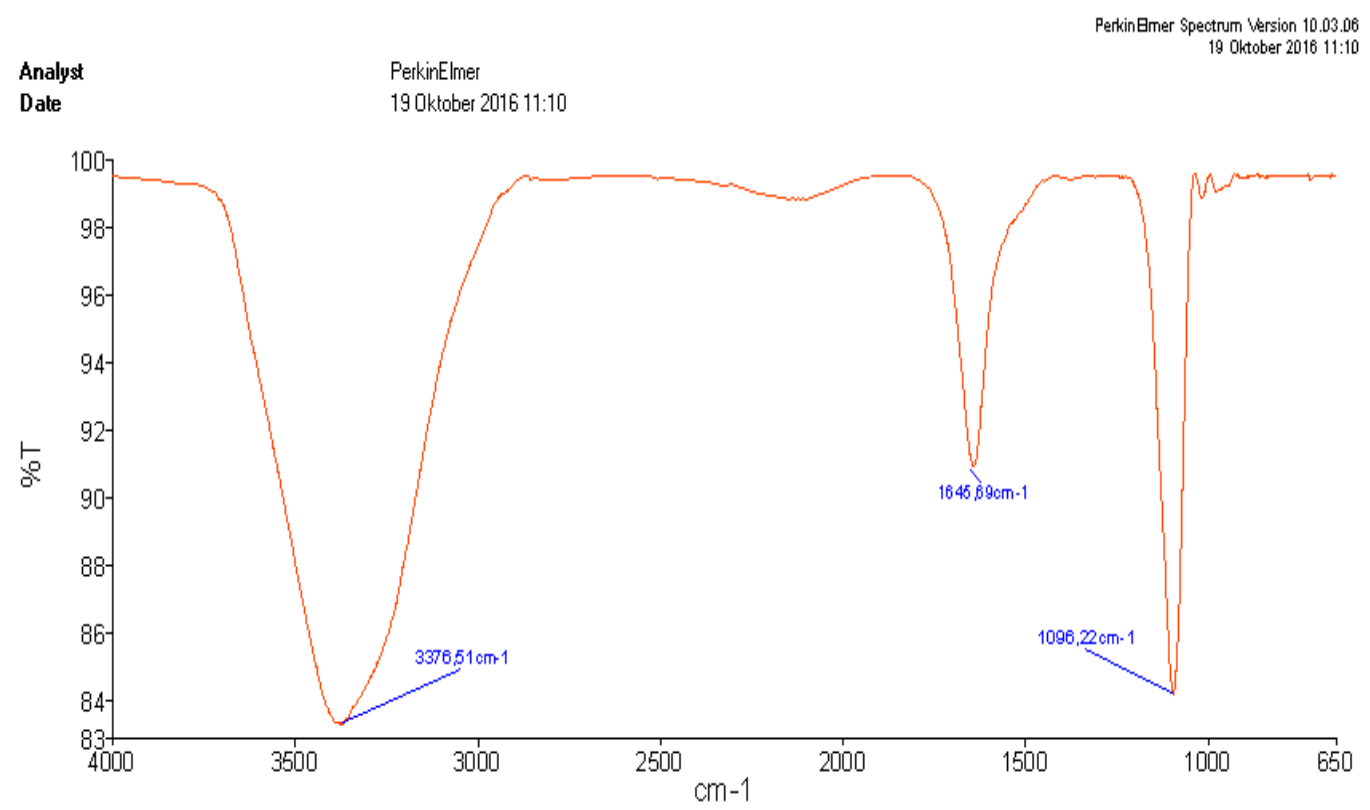

Fig. 3. FTIR spectra of prepared alumina before washing sulfate ion.

The Fourier transport infra-red spectra (FTIR) shown from Fig. 3, the typical peak in the FTIR spectra were obtained after observed in the region comprised of bands assigned to the pure region to alumina. The peaks are shown $3376.5 \mathrm{~cm}^{-1}, 1645 \mathrm{~cm}^{-1}, 1096.22 \mathrm{~cm}^{-1}$ stretching of alumina-alumina bond. The ion stretching region is particularly useful for elucidating alumina-bonding patterns. At wave numbers between 1000 and $3500 \mathrm{~cm}-1$ the intensities decreased due to the washing. This peak has been assigned to the asymmetrical stretching 
of alumina. As the washing of sulfate ions was done, the Fourier transport infra-red spectra (FTIR) in shown in Fig. 4, the typical peak in the FTIR spectra were obtained after observed in the region comprised of bands assigned to the pure region to alumina. The peaks are shown $3376.5 \mathrm{~cm}^{-1}, 1645 \mathrm{~cm}^{-1}, 1096.22 \mathrm{~cm}^{-1}, 1422 \mathrm{~cm}^{-1}$ and $1030 \mathrm{~cm}^{-1}$ this indicate that alumina getting pure and stretching of alumina-alumina bond exist. The ion stretching region is particularly useful for elucidating alumina-bonding patterns. The typical peak for pure alumina is expected between 1000 and $3500 \mathrm{~cm}-1$ the intensities decreased due to the washing. This peak has been assigned to the asymmetrical stretching of alumina.

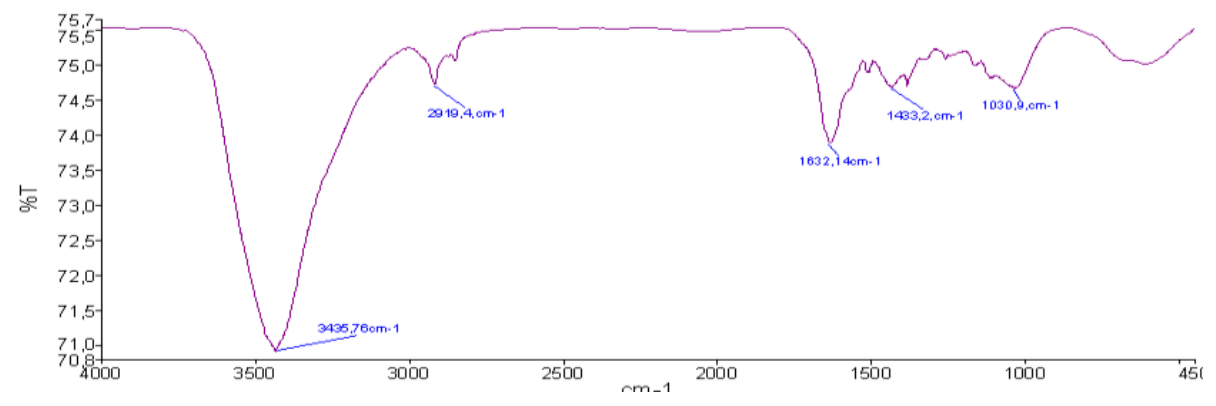

Fig.4. FTIR spectra of prepared alumina after washing sulfate ion.

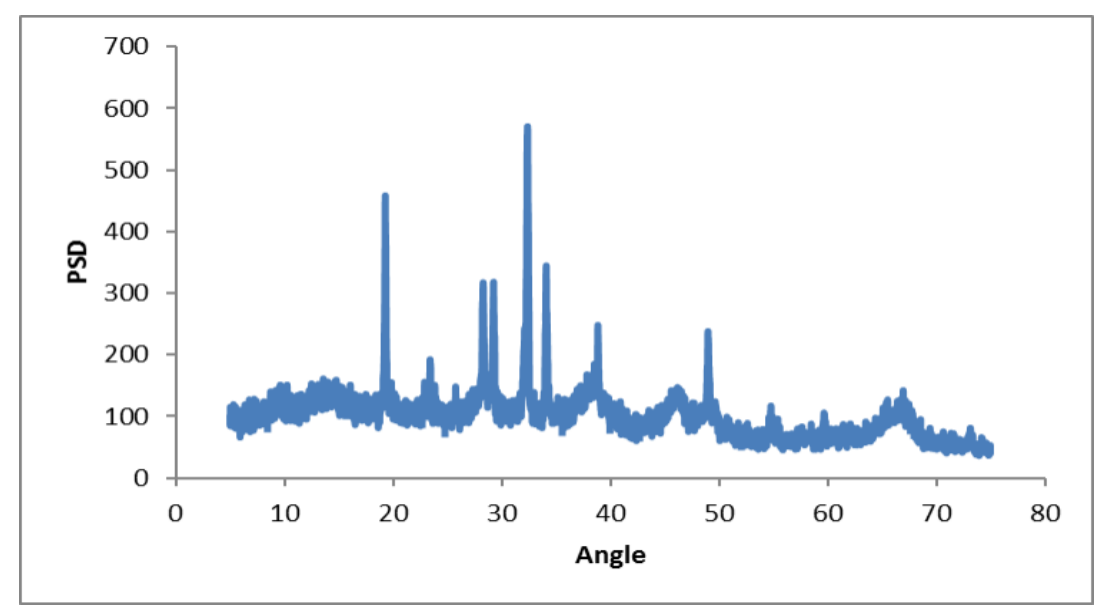

Fig.5. XRD patterns of alumina particles.

Fig. 5 shows the XRD patterns of the $\mathrm{ZnO}$ after washed different water flow rate. Generally can be observed that main peaks observed in the sample which locate at $2 \theta=$ 18.76, 23.51 and 33.885 which can be attributed to the active alumina bond at xyz, (100) and (200) crystal planes of the alumina active phase of alumina. It is important to note that the X-ray diffraction patterns of alumina particle over the entire purification range show no additional peaks exist for corresponding to secondary impurities such as sulfate ions and also other closely related cation-ordered orthorhombic structures of other elements such radicals. The intensity of the 35 peak becomes more intense and sharp, with the increase wash rate, the diffraction peaks monotonously shifts towards small angles as can be observed indicating that the lattice constant increases which in other attributed for being getting pure. 


\section{Conclusions}

The study demonstrated the preparation and characterization of activated aluminum via pure aluminum $(99.9 \%$ pure), which subsequently reacted at room temperature, with a aqueous $\mathrm{NaOH}$ in a reactor to produce a solution of sodium aluminate $\left(\mathrm{NaAlO}_{2}\right)$. This solution was passed through filter paper and the clear filtrate was neutralized with $\mathrm{H}_{2} \mathrm{SO}_{4}$, to $\mathrm{pH} 6,7$ or 8 , resulting in the precipitation of a white gel, $\mathrm{Al}(\mathrm{OH})_{3} \cdot \mathrm{XH}_{2} \mathrm{O}$. The washed gel for sulfate ions were dried at $80{ }^{\circ} \mathrm{C}$ for $6 \mathrm{~h}$, a 60 mesh sieve was to separate and sort them into different sizes. The samples were then calcined (burn) for $3 \mathrm{~h}$ in a muffle furnace, in air, at a heating rate of $2{ }^{\circ} \mathrm{C} \mathrm{min}^{-1}$. The characterization of the activated alumina revealed that the activated alumina is obtained. The approach is simple yet could produce pure activated alumina.

\section{References}

1. S. Ahmed, M.G. Rasul, W.N. Martens, R. Brown, M.A. Hashib. Desalination 261, 318 (2010)

2. D. Musmarra, D. Karatza, A. Lancia, M. Prisciandaro, G.M. di Celso, Chem. Eng. Trans. 32, 547-552 (2013)

3. T. Anirudhan, S. Sreekumari, J. Environ. Sci. 23, 1989-1998 (2011)

4. Y. Guo, E. Du, Energy Procedia. 17, 444-449 (2012)

5. F. Salvador, N. Martin-Sanchez, R. Sanchez-Hernandez, M.J. Sanchez-Montero, C. Izquierdo, Microporous Mesoporous Mater. 202, 259-276 (2015)

6. F. Salvador, N. Martin-Sanchez, R. Sanchez-Hernandez, M.J. Sanchez-Montero, Microporous Mesoporous Mater. 202, 277-296 (2015)

7. S. Ahmed, M.G. Rasul, R. Brownb, M.A. Hashib. Journal of Environmental Management 92, 311-330 (2011)

8. U. Beker, B. Ganbold, H. Dertli, D. Duranog, I. Gulbayir. Energy Conversion and Management 51, 235-240 (2010)

9. L. Cadoret, C. Rossignol, J. Dexpert-Ghys, et al. Materials Science and Engineering BAdvanced Functional Solid- State Materials 170 (1-3), 41-50 (2010)

10. S.A. Darko, E. Maxwell, S. Park, Thin Solid Films 519(1), 174-177 (2010)

11. S.M. Lam, J.C. Sin, A.R. Mohamed, Korean J. Chem. Eng. 27(4), 1109-1116 (2010)

12. C. Saka, Journal of Analytical and Applied Pyrolysis 95, 21-24 (2012)

13. I.K. Shah, P. Pre, B. Alappat, Journal of the Taiwan Institute of Chemical Engineers, 45, 1733-1738 (2014)

14. Xin-hui, Process Intensification 53, 53- 62 (2012)

15. M. Barakat, Arabian Journal of Chemistry 4, 361-377 (2011)

16. R. Berenguer, J.P. Marco-Lozar, C. Quijada, D. Cazorla-Amorós, E. Morallón, Carbon 48, 2734-2745 (2010)

17. A. Eliassi, M. Ranjbar, Int. J. Nanosci. Nanotechnol. 10, 1, 13-26 (2014).

18. B. Ksapabutr, E. Gulari, S. Wongkasemjit, Physicochem. Eng. Aspects 233 145-153 (2004)

19. M.A. Karakassides, D. Gournis, A.B. Bourlinos, P.N. Trikalitis, T.B. Magneti, J. Mater. Chem. 13, 871-876 (2016)

20. P.R. Reddy, K.M. Ajith, N.K. Udayashankar, Adv. Mater. Lett. 7(5), 398-401(2016)

21. G. Karthikeyan', B.V. Apparao, S. Meenakshi, Defluoridation properties of activated alumina (2nd international workshop on fluorosis prevention and defluoridation of water, 79-82, 2014) 